\title{
Pyk2 activation is integral to acid stimulation of sodium/hydrogen exchanger 3
}

\author{
Shaoying Li, Soichiro Sato, Xiaojing Yang, Patricia A. Preisig, and Robert J. Alpern \\ Department of Internal Medicine, University of Texas Southwestern Medical Center, Dallas, Texas, USA.
}

\begin{abstract}
The present study examines the role of Pyk2 in acid regulation of sodium/hydrogen exchanger 3 (NHE3) activity in OKP cells, a kidney proximal tubule epithelial cell line. Incubation of OKP cells in acid media caused a transient increase in Pyk2 phosphorylation that peaked at 30 seconds and increased Pyk2/c-Src binding at 90 seconds. Pyk2 isolated by immunoprecipitation and studied in a cell-free system was activated and phosphorylated at acidic $\mathrm{pH}$. Acid activation of Pyk2 (a) was specific for Pyk2 in that acid did not activate focal adhesion kinase, (b) required calcium, and (c) was associated with increased affinity for ATP. Transfection of OKP cells with dominant-negative $p y k 2^{\mathrm{K} 457 \mathrm{~A}}$ or small interfering pyk2 duplex RNA blocked acid activation of NHE3, while neither had an effect on glucocorticoid activation of NHE3. In addition, $p y k 2^{\mathrm{K} 457 \mathrm{~A}}$ blocked acid activation of c-Src kinase, which is also required for acid regulation of NHE3. The present results demonstrate that Pyk2 is directly activated by acidic $\mathrm{pH}$ and that Pyk2 activation is required for acid activation of c-Src kinase and NHE3. Given that partially purified Pyk2 can be activated by acid in a cell-free system, Pyk2 may serve as the $\mathrm{pH}$ sensor that initiates the acid-regulated signaling cascade involved in NHE3 regulation.
\end{abstract}

\section{Introduction}

Metabolic acidosis induces a series of homeostatic responses in various organs that serve in a concerted manner to return extracellular $\mathrm{pH}$ toward normal. These processes include increased acid secretion in the kidney, increased renal ammonia synthesis, increased osteoclast-mediated bone resorption, and muscle protein degradation (1). In the renal proximal tubule, metabolic acidosis induces increased rates of hydrogen secretion into the luminal fluid, citrate reabsorption from the luminal fluid, and ammonia synthesis. These processes involve regulation of multiple enzymes and transporters. Previous studies have demonstrated that this concerted response in the proximal tubule is mediated by an acid-regulated signaling pathway that includes activation of c-Src and ERK and increases in c-fos, c-jun, junB, and egr-1 expression (1-5).

Using maneuvers such as weak acid addition and $\mathrm{NH}_{3}$ prepulse that induce intracellular acidification in the absence of extracellular acidification, researchers have demonstrated that it is intracellular acidification that increases c-Src activity (5). However, the mechanism by which this occurs has not been elucidated. Presumably, the cell possesses a $\mathrm{pH}$-sensitive protein, or $\mathrm{pH}$ sensor, that is activated by decreases in intracellular $\mathrm{pH}$ and then initiates a signaling cascade involving the above pathways. Because c-Src is activated by acid, we first examined $\mathrm{p} 125^{\mathrm{FAK}}$ as a possible upstream activator. However, although $\mathrm{p} 125^{\mathrm{FAK}}$ is activated in OKP cells by media acidification, activation occurs at 6 hours, which is later than c-Src activation (30-90 seconds) $(5,6)$.

In the present study, we examined the role of Pyk2, a member of the focal adhesion kinase (FAK) family of tyrosine kinases (7). Pyk2 is activated in OK cells by cholinergic agents, angiotensin II,

Nonstandard abbreviations used: BCECF, 2'7'-bis (2-carboxyethyl)-5-(and-6)-carboxyfluorescein; FAK, focal adhesion kinase; MEK, mitogen-activated protein/ERK kinase; NaDC-1, sodium-dicarboxylate cotransporter 1; NBC-1, sodium bicarbonate cotransporter 1; NHE3, sodium/hydrogen exchanger 3; PEPCK, phosphoenolpyruvate carboxykinase; $\mathrm{pHi}$, cytoplasmic $\mathrm{pH}$; siRNA $\mathrm{pyk}_{2}$, small interfering pyk2 RNA.

Conflict of interest: The authors have declared that no conflict of interest exists.

Citation for this article: J. Clin. Invest. 114:1782-1789 (2004).

doi:10.1172/JCI200418046. and $\mathrm{CO}_{2}$ and plays a role in sodium bicarbonate cotransporter 1 (NBC-1) regulation (8). In multiple systems, Pyk2 activates $\mathrm{c}$-Src and is activated by calcium. The results demonstrate that (a) media acidification activates Pyk2 in whole cells, (b) decreases in $\mathrm{pH}$ can activate Pyk 2 in a cell-free system, and (c) Pyk2 activation is specific and required for acid activation of both c-Src kinase and sodium/hydrogen exchanger 3 (NHE3).

\section{Results}

Acid incubation induces Pyk2 phosphorylation in OKP cells. To examine the role of Pyk2 in the cellular response to acidosis, we first examined the effect of media acidification on Pyk 2 phosphorylation in OKP cells. Activated Pyk 2 autophosphorylates at $\mathrm{Tyr}^{402}$ and activates c-Src, which then further phosphorylates Pyk2 on several additional sites (7). Pyk2 phosphorylation thus provides an accurate assay for Pyk2 activation (7).

OKP cells were incubated at $\mathrm{pH} 7.4$ or 6.8 , Pyk2 was immunoprecipitated, and Pyk2 phosphorylation was assessed. As shown in Figure 1, incubation in acid media caused a transient increase in Pyk 2 phosphorylation, which peaked at 30 seconds.

Pyk 2 may be activated secondary to activation of another signaling molecule or could be activated directly by acid intracellular $\mathrm{pH}$ and thus would represent the $\mathrm{pH}$ sensor. To determine if $\mathrm{Pyk} 2$ can be directly activated by acid, we isolated Pyk2 from cell lysates by immunoprecipitation, incubated the Pyk 2 at varying $\mathrm{pH}$ values, and assayed Pyk 2 activity by its ability to phosphorylate a synthetic substrate [poly(Glu-Tyr ${ }^{4: 1}$. Incubation at $\mathrm{pH} 7.0$ or 6.8 for 5 minutes increases Pyk2 phosphorylation of poly(Glu-Tyr $)^{4: 1}$ to $304 \%$ and $288 \%$, respectively, of control (Figure 2). These results demonstrate that acid $\mathrm{pH}$ can directly activate $\mathrm{Pyk} 2$ in the absence of cell contents and suggest that $\mathrm{Pyk} 2$ is directly regulated by proton concentration.

As noted above, activated Pyk2 autophosphorylates on Tyr ${ }^{402}$. Therefore, we measured Pyk2 autophosphorylation following acid incubation in vitro. Similar to acid stimulation of Pyk2 kinase activity, incubation in a cell-free system at $\mathrm{pH} 7.2,7.0$, or 6.8 for 5 minutes caused Pyk2 autophosphorylation to increase to $205 \%$ of control (Figure 3 ). 


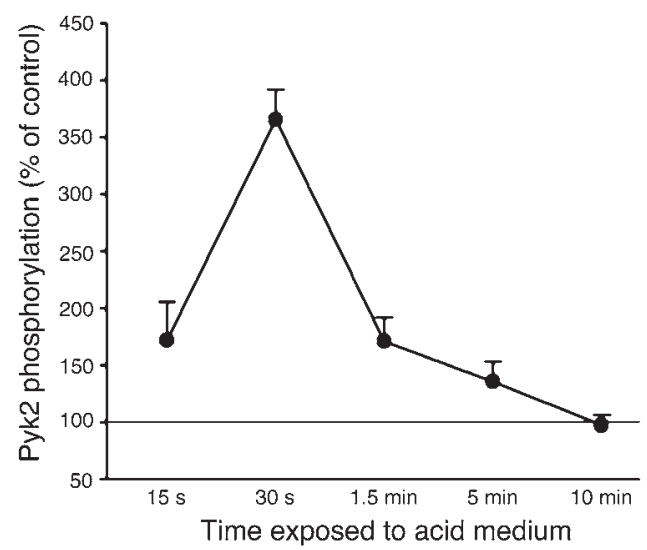

Figure 1

Acid incubation induces Pyk2 phosphorylation in OKP cells. After growing to confluence, cells were rendered quiescent for 48 hours and then exposed to a medium of $\mathrm{pH} 7.4$ (control) or 6.8 (acid) for the indicated time. Pyk2 was then immunoprecipitated and phosphorylation assayed, as described in Methods. Data are plotted as percentage of control; $n=6$ for control and acid at each time point. Pyk2 phosphorylation was increased in 4 of 6 experiments ( 15 seconds), 6 of 6 experiments ( 30 seconds and 5 minutes), 5 of 6 experiments (90 seconds), and 2 of 6 experiments (10 minutes).

Acid incubation does not activate FAK. To determine if activation by acid is specific for Pyk2, studies were performed with FAK, a member of the same family of tyrosine kinases as Pyk2 (7). Although we have previously shown in whole cells that media acidification activates FAK, this effect required 6 hours of media acidification and thus it is unlikely that acid activation of FAK is a direct effect of acid. As shown in Figure 4, incubation in acid $\mathrm{pH}$ did not increase poly(Glu-Tyr $)^{4: 1}$ phosphorylation by FAK, demonstrating that the direct effect of acid on Pyk2 activity is specific.

Calcium is required for acid activation of Pyk2. In many systems, Pyk2 activation is $\mathrm{Ca}^{2+}$ dependent. In the studies described above, $\mathrm{Ca}^{2+}$ was not added to the buffer solution; however, there is probably a small amount of $\mathrm{Ca}^{2+}$ in deionized water. To determine if $\mathrm{Ca}^{2+}$ is required for acid activation of Pyk2, activation was studied in the presence and absence of $1 \mathrm{mM}$ EGTA. The presence of EGTA not only blocked acid activation of Pyk 2 kinase but caused acid-induced inhibition of Pyk2 activity (ratio $\mathrm{pH} 6.8 / \mathrm{pH} 7.4=0.65 \pm 0.05$, $n=5$ ). These data demonstrate a $\mathrm{Ca}^{2+}$ dependence of Pyk 2 activation by acid and suggest that, in the absence of $\mathrm{Ca}^{2+}$, acid inhibits Pyk2 kinase activity.

Acid activation of Pyk2 kinase is associated with an increased affinity to ATP. To begin to elucidate the mechanism of acid regulation of Pyk2 kinase activity, studies were performed in which kinase activity was

\section{Figure 2}

Acid activates Pyk2 in a cell-free system. Wild-type OKP cells were grown to confluence and rendered quiescent for 48 hours. Pyk2 was immunoprecipitated as described in Methods and exposed to buffers at the indicated $\mathrm{pH}$ for 5 minutes; kinase activity was assayed as the ability of Pyk2 to phosphorylate poly(Glu-Tyr) $)^{4: 1}$. (A) Typical autoradiograph (top) and Western blot (bottom). (B) Summary of the data, which are normalized for Pyk2 abundance and plotted as a percentage of control $(\mathrm{pH} 7.4) ; n=9$ for each $\mathrm{pH}$. Pyk2 kinase activity was increased in 8 of 9 experiments at $\mathrm{pH} 7.2,7.0$, and 6.8.

assayed at $\mathrm{pH} 7.4$ and 6.8 in the presence of varying concentrations of $\operatorname{ATP}(5,20,100$, and $200 \mu \mathrm{M})$. Acid incubation did not affect maximal kinase activity, but rather the affinity for ATP increased. Using an Eadie-Hofstee analysis, the calculated $K_{\mathrm{mATP}}$ was $129 \pm 21 \mu \mathrm{M}$ at $\mathrm{pH} 7.4$ and $51 \pm 6 \mu \mathrm{M}$ at $\mathrm{pH} 6.8(P<0.03, n=4)$. The calculated $V_{\max }$ was $0.182 \pm 0.111$ at $\mathrm{pH} 7.4$ and $0.246 \pm 0.138$ at $\mathrm{pH} 6.8(\mathrm{NS}, n=4)$.

Dominant-negative Pyk2 prevents NHE3 activation by acid. Subsequent studies used 2 approaches to determine the role of Pyk2 in acid activation of NHE3. First, OKP cells were transiently transfected with dominant-negative pyk2 ( $\left.p y k 2^{\mathrm{K} 457 \mathrm{~A}}\right)(7)$. Mutation of lysine 457 to alanine in the tyrosine-kinase domain of Pyk2 creates an inactive Pyk2 that inhibits wild-type Pyk2 activity $(7,9)$. Cells were incubated at $\mathrm{pH} 6.8$ or 7.4 for 6 hours, and NHE3 activity was measured using 2'7'-bis (2-carboxyethyl)-5-(and-6)-carboxyfluorescein (BCECF). As shown in Figure 5, in wild-type OKP cells, incubation in acid media caused a $41 \%$ increase in NHE3 activity $(P<0.01)$. This response is similar to that previously reported (10). Similarly, in cells transiently transfected with vector alone or wild-type pyk2, acid incubation caused $25 \%$ and $21 \%$ increases, respectively, in NHE3 activity $(P<0.01$, Figure 5). However, in cells transfected with dominant-negative $p y k 2^{\mathrm{K} 457 \mathrm{~A}}$, acid incubation had no effect on NHE3 activity ( $3 \%$ increase, NS, Figure 5).

To demonstrate the specificity of the dominant-negative Pyk2 effect, we examined its effect on glucocorticoid activation of NHE3. NHE3 activation by glucocorticoids does not involve tyrosine-kinase pathways and thus should not be blocked by inhibition of Pyk2. We have previously shown that inhibition of c-Src or mitogen-activated protein/ERK kinase (MEK) prevents acid activation of NHE3 but not activation by glucocorticoids $(2,4)$. Cells were incubated with $10^{-7} \mathrm{M}$ dexamethasone or vehicle for 24 hours. In cells transfected with dominant-negative $p y k 2^{\mathrm{K} 457 \mathrm{~A}}$, dexamethasone increased NHE3 activity by $48 \%(P<0.01, n=6)$, a degree of stimulation similar to that seen in wild-type OKP cells (11).

Small interfering pyk2 RNA prevents NHE3 activation by acid. As a second approach, we decreased Pyk2 expression in OKP cells using small interfering pyk2 RNA (siRNA pyk2 $\left._{2}\right)(12,13)$. Because siRNAs require a perfect sequence match with their target $\mathrm{mRNAs}$, we first cloned a partial sequence of the OKP pyk2. To accomplish this, PCR primers were generated as described in Methods based on pyk2 sequences conserved in human and rat. The PCR product generated was 405 $\mathrm{bp}$, the predicted size, and when sequenced yielded a pyk2 sequence that was $88 \%$ identical to human and rat pyk2 and $85 \%$ identical to mouse pyk2 (GenBank accession numbers: opossum, AY195883; human, GI1245923; rat, GI1000679; and mouse, GI5905541). We then used an internal sequence to generate an siRNA that corre-

A

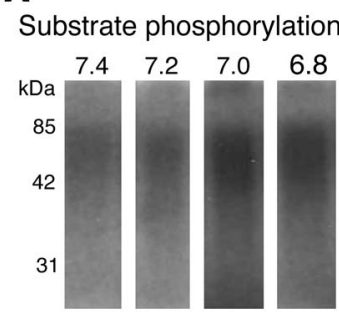

Pyk2 abundance
B

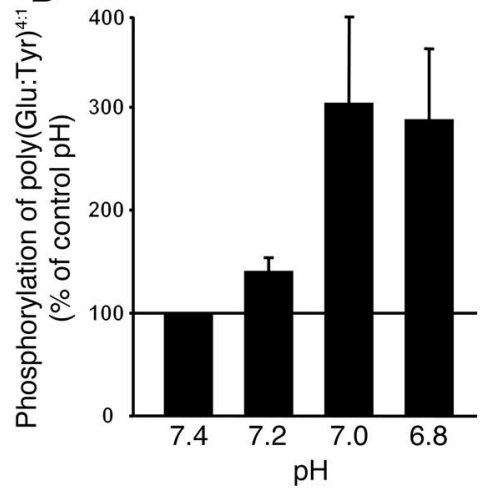




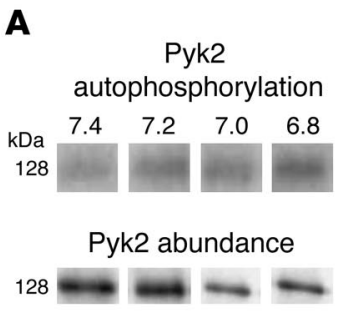

\section{Figure 3}

Acid induces Pyk2 autophosphorylation in a cell-free system. Wild-type OKP cells were grown to confluence and rendered quiescent for 48 hours. Pyk2 was immunoprecipitated as described in Methods and exposed to buffers at the indicated $\mathrm{pH}$ for 5 minutes; Pyk2 autophosphorylation was assayed. (A) Typical autoradiograph (top) and Western blot (bottom). (B) Summary of the data, which are normalized for Pyk2 abundance and plotted as a percentage of control $(\mathrm{pH} 7.4) ; n=9$ for each pH. Pyk2 phosphorylation was increased in 5 of 9 experiments at $\mathrm{pH} 7.2$ and 7.0 and in 8 of 9 experiments at $\mathrm{pH} 6.8$.

sponded to a coding sequence, was distal to the first 100 bases of the coding sequence, and had a GC content of approximately $50 \%$. The sequence used was 5'-(AA)UGCCCUUGAUAAGAAGUCC-3', corresponding to nucleotides 325-343 of the cloned opossum $p y k 2$ and nucleotides 872-890 (or 579-597 relative to the start codon) of the human pyk2 nucleotide sequence.

Transfection with siRNA $A_{\text {pyk } 2}$ caused a 90\% decrease in Pyk 2 protein abundance as assessed by immunoblot (Figure 6). As shown in Figure $6 \mathrm{~A}$, the expression of Pyk 2 protein was specifically decreased by transfection with ${ }^{2} \mathrm{RNA}_{\text {pyk2 } 2}$ duplex but not by transfection with a nonspecific siRNA ( siRNA $_{\mathrm{GL} 2}$ ). Expression of a nontarget gene, FAK, which belongs to the same family as Pyk2, was not affected. Silencing was monitored at different time points after transfection

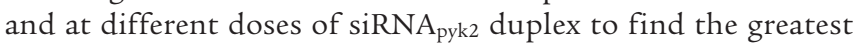
effect on Pyk2 protein abundance. As shown in Figure 6, B and C, Pyk 2 was silenced within 24 hours of beginning transfection and remained silenced after 72 hours. The largest effect was seen at siRNA $A_{\text {pyk } 2}$ doses equal to or greater than $1.33 \mu \mathrm{g}$ per well.

In control cells not treated with siRNA, acid incubation caused a $30 \%$ increase in NHE3 activity $(P<0.05$, Figure 7$)$. Similarly, in cells transfected with siRNA $\mathrm{GL}_{2}$, acid incubation caused a $28 \%$ increase in NHE3 activity $(P<0.05)$. However, in cells transfected with siRNA $A_{\text {pyk2 }}$, acid incubation had no effect on NHE3 activity ( $1 \%$ increase, NS), again demonstrating that Pyk 2 is required for NHE3 activation (Figure 7).

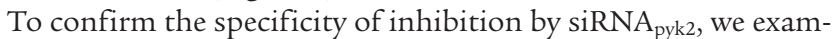
ined the effect of glucocorticoids on NHE3 activity. In cells

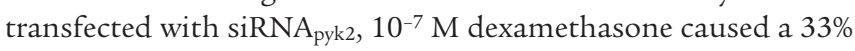
increase in NHE3 activity $(P<0.05, n=5)$. These results demonstrate that the effect of $\mathrm{Pyk} 2$ inhibition on NHE3 activation is specific for the acid-activated signaling pathway.

Acid incubation increases Pyk2 and c-Src binding, which is necessary for acid stimulation of NHE3 activity. Following activation, Pyk2 autophosphorylates and then binds to and activates c-Src, which in turn further phosphorylates Pyk2 (14). We have previously shown that c-Src is necessary for acid regulation of NHE3 activity $(2,4,5)$. To examine whether acid incubation increases binding of c-Src to Pyk2, coimmunoprecipitation studies were performed following incubation in a control ( $\mathrm{pH} 7.4$ ) or acid ( $\mathrm{pH}$ 6.8) medium. By 90 seconds, the peak time of c-Src activation (5), there was a $107 \%$ increase in c-Src abundance in anti-Pyk 2 immunoprecipitates and a $115 \%$ increase in Pyk2 abundance in anti-v-Src immunoprecipitates harvested from cells incubated in acid, compared to control media (Figure 8).

Grb2 is an adaptor molecule with a putative binding site on Pyk2 (tyrosine 881) (14-17). Next we examined whether acid increased Grb2 binding to Pyk2. Results showed that Grb2 does coprecipitate with Pyk 2 but that there was no effect of acid on coprecipitated Grb2 abundance at 30 and 90 seconds or at 2 minutes. At $60 \mathrm{sec}-$ onds, acid significantly decreased the abundance of coprecipitated Grb2 $($ acid/control ratio $=0.69 \pm 0.09, P<0.04, n=4)$.

To determine whether acid-induced c-Src binding to Pyk 2 is necessary for acid regulation of NHE3 activity, we examined the effect of the Pyk 2 mutant Pyk2 $2^{\mathrm{Y} 402 \mathrm{~F}}$ on acid regulation of NHE3 activity. Mutation of tyrosine 402 to phenylalanine prevents binding of c-Src to activated Pyk2, and subsequent Src activation (9). In vector transfected cells, acid incubation increased NHE3 activity $24 \% \pm 5 \%$ $(n=6, P<0.03)$ whereas in $p y k 2^{Y 402 F}$ transfected cells acid was without effect on NHE3 activity ( $4 \% \pm 6 \%$ increase, NS, $n=6)$. Thus Pyk2 autophosphorylation and c-Src binding to Pyk2 are necessary for acid stimulation of NHE3 activity.

Pyk2 is required for acid activation of $c$-Src. Having established that acid activates both Pyk2 and c-Src kinases and that both kinases and Pyk2/c-Src binding are required for acid regulation of NHE3, we next addressed the question of whether Pyk 2 activation is required for acid activation of c-Src. For these studies, cells were transiently transfected with c-Src and either wild-type or dominant-negative Pyk2. Following incubation in control or acid media, c-Src kinase activity was assayed using acid-denatured enolase as the substrate. As shown in Figure 9, expression of the dominantnegative Pyk2 prevented acid activation of c-Src kinase.

\section{Discussion}

Metabolic acidosis induces a diverse response in proximal tubule cells, including increases in the activities of NHE3, NBC-1, glutaminase, glutamate dehydrogenase, ATP citrate lyase,
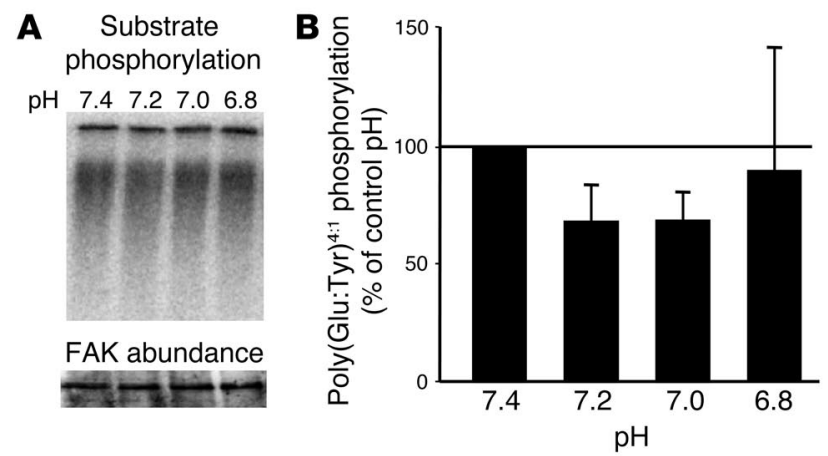

\section{Figure 4}

Acid does not activate FAK in a cell-free system. OKP cells were grown to confluence and rendered quiescent for 48 hours. FAK was immunoprecipitated as described in Methods and exposed to buffers at the indicated $\mathrm{pH}$ for 5 minutes; kinase activity was assayed as the ability of FAK to phosphorylate poly(Glu-Tyr) ${ }^{4: 1}$. (A) Typical autoradiograph (top) and Western blot (bottom). (B) Summary of the data, which are normalized for FAK abundance and plotted as a percentage of control $(\mathrm{pH} 7.4) ; n=3$ for $\mathrm{pH} 7.4,7.2$, and 7.0, and $n=2$ for $\mathrm{pH} 6.8$. 


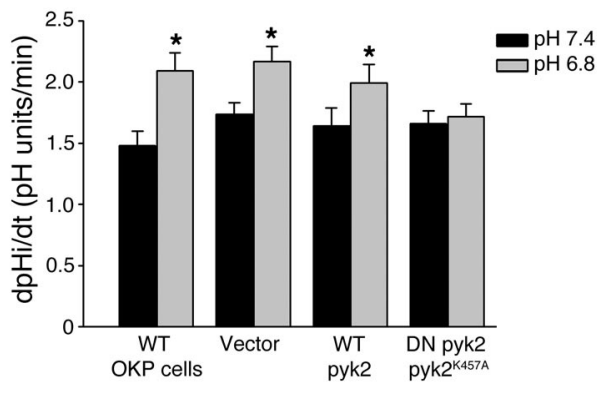

Figure 5

Dominant-negative Pyk2 prevents NHE3 activation by acid. OKP cells were transiently transfected with $1 \mu \mathrm{g}$ of the indicated DNA for 5 hours, grown to confluence over the next 19 hours, rendered quiescent for 24 hours, and exposed to control $(\mathrm{pH} 7.4)$ or acid $(\mathrm{pH} \mathrm{6.8)} \mathrm{media} \mathrm{for}$ 6 hours; $\mathrm{Na} / \mathrm{H}$ antiporter activity was then assayed as the rate of $\mathrm{Na}$ dependent increase in cell $\mathrm{pH}$. Data are plotted as dpHi/dt, $\mathrm{pH}$ units/ $\min ; n=6$ (wild-type cells), $n=19$ (vector transfected), $n=19$ (wildtype Pyk2 transfected), and $n=17$ (dominant-negative [DN] Pyk2 ${ }^{\mathrm{K} 457 \mathrm{~A}}$ transfected). ${ }^{*} P<0.01$ vs. $\mathrm{pH} 7.4$.

mitochondrial aconitase, sodium-dicarboxylate cotransporter 1 (NaDC-1) and phosphoenolpyruvate carboxykinase (PEPCK) (1). Increases in the activities of these enzymes and transporters results in increased rates of $\mathrm{H}^{+}$secretion, citrate reabsorption, and ammonia synthesis, all of which participate in processes that return blood $\mathrm{pH}$ to normal. This coordinated response is due to activation of a signaling cascade that includes activation of cSrc and ERKs and increases in the expression of $c$-fos, c-jun, junB, and egr-1 (2-5). In addition, this response involves an increase in preproendothelin- 1 gene expression, with secretion of endothelin 1 and activation of the endothelin B receptor $(18,19)$.

Although the above studies have defined components of a signaling cascade, they have not defined the protein that is directly activated by decreases in intracellular $\mathrm{pH}$ and that initiates the cascade. In an attempt to identify a possible $\mathrm{pH}$ sensor/activator of c-Src, we first examined p125 $5^{\mathrm{FAK}}$. While $\mathrm{p} 125^{\mathrm{FAK}}$ was activated by acid in whole cells, its time course was delayed compared with cSrc activation, and thus it cannot be upstream $(5,6)$. Pyk 2 is a nonreceptor tyrosine kinase that was first identified by Lev et al. (7). It is closely related to $\mathrm{p} 125^{\mathrm{FAK}}$ and is also able to activate c-Src.

The present studies examined the role of Pyk2 in the acid signaling cascade that regulates NHE3 activity. We first found that exposing OKP cells to acid media transiently activated Pyk2. Peak Pyk 2 activation occurred at 30 seconds, before peak c-Src activation at 30-90 seconds (5). This could be a direct effect of $\mathrm{pH}$ on Pyk 2 or could be secondary to activation of another protein or intermediate. To address this, we isolated Pyk 2 from cell lysates by immunoprecipitation, resuspended the precipitate, and exposed it to varying $\mathrm{pHs}$ for 5 minutes. Incubation at $\mathrm{pH}$ 6.8-7.2 consistently increased Pyk2 activity, assayed as the ability of Pyk 2 to phosphorylate a synthetic substrate, and was associated with Pyk2 autophosphorylation. Activation by acid $\mathrm{pH}$ was specific for Pyk2 and was not seen with FAK. While all proteins have a $\mathrm{pH}$ optima, a $\mathrm{pH}$ sensor is a protein whose activity varies considerably over a smaller physiologically relevant range (e.g., pH 6.8-7.4 for Pyk2). Although these results suggest that Pyk 2 is directly activated by decreases in $\mathrm{pH}$, it is possible that the immunoprecipitate also contains a $\mathrm{Pyk} 2$ binding protein that is the $\mathrm{pH}$ sensor.
It should be noted that the degree of Pyk2 phosphorylation observed in the cell-free system (Figure 3) was increased to only $205 \%$ of control whereas Pyk 2 phosphorylation increased to $365 \%$ of control in the intact cell upon acid activation (Figure 1). This difference was present even though the degree of acidification was likely greater in the cell-free system than would be achieved in the intact cell. This is likely due to the fact that in the intact cell Pyk2 is autophosphorylated and then further phosphorylated and activated by c-Src. When Pyk2 is activated in the cell-free system, c-Src is not present and thus only autophosphorylation occurs.

Our studies show that Pyk2 kinase activity can be regulated by $\mathrm{pH}$ in a cell-free system and that this involves an increase in the affinity of the kinase for ATP. Given that measured whole-cell ATP concentrations are in the millimolar range and the affinity for ATP is in the micromolar range, a change in the Michaelis-Menten constant for ATP would not seem to be physiologically relevant. However, Mandel and coworkers $(20,21)$ demonstrated that, although the isolated proximal tubule Na,K-ATPase has a Michaelis-Menten constant for ATP in the range of 300-500 $\mu \mathrm{M}$ and cellular ATP concentrations are in the millimolar range, maneuvers that decrease cellular ATP content by $30-45 \%$ in the intact tubule inhibit the Na,K-ATPase transport rate by $30-45 \%$. These studies suggest that in the intact tubule, the Na,K-ATPase functions at the linear part of the ATP dependence curve. Thus it is possible that local ATP concentrations within microdomains of ATPases or kinases are lower than those measured in whole-cell lysates or that the affinity of these ATPases/kinases for ATP is lower than that measured on the isolated protein, possibly due to the presence of other regulatory molecules in the intact cell.

We have previously shown that inhibition of c-Src or MEK prevents acid activation of NHE3 $(2,4)$. In the present study, we used three approaches to demonstrate the role of Pyk 2 in acid regulation of NHE3 and to determine if Pyk2 is necessary for acid signaling through c-Src. Pyk 2 activation and Pyk $2 / \mathrm{c}-\mathrm{Src}$ binding are initiated by $\mathrm{Pyk} 2$ autophosphorylation on $\mathrm{Tyr}^{402}$, which leads to binding of the c-Src SH2 domain to a motif that includes the $\mathrm{Tyr}^{402}$ residue, with subsequent c-Src activation (9). Src activation leads to further Pyk2 phosphorylation in the kinase and C-termi-

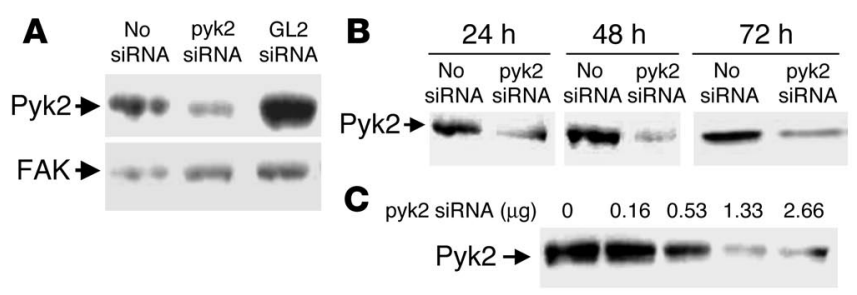

Figure 6

siRNA $A_{\text {pyk2 }}$ decreases Pyk2 protein abundance in OKP cells. Typical blots. (A) Cells were transfected with $1.33 \mu \mathrm{g} / \mathrm{well}$ siRNA $A_{\text {pyk2 }}$ or siRNA $\mathrm{GL}_{2}$ (GL2 siRNA) or transfection reagent only (No siRNA) for 24 hours (at which time they were confluent), rendered quiescent for 24 hours, and harvested. FAK protein abundance was measured for comparison. (B) Cells were transfected with $1.33 \mu \mathrm{g} /$ well siRNA $_{\text {pyk2 }}$ for 24 hours (at which time they were confluent) and rendered quiescent. The length of quiescence varied from 0 to 48 hours, so that cells were harvested 24,48 , or 72 hours after the beginning of transfection. (C) Cells were transfected with the indicated amount of siRNA $A_{\text {pyk } 2}$ for 24 hours (at which time they were confluent), rendered quiescent for 24 hours, and harvested. 


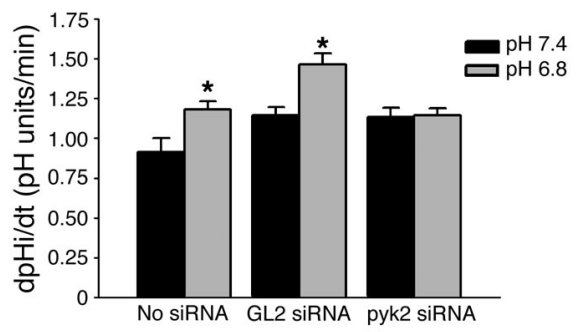

Figure 7

siRNA $A_{\text {pyk2 }}$ prevents NHE3 activation by acid. OKP cells were transiently transfected with $1.33 \mu \mathrm{g}$ siRNA $A_{\text {pyk2 }}$ (pyk2 siRNA) per well for 24 hours (at which time they were confluent), rendered quiescent for 18 hours, and exposed to control $(\mathrm{pH} \mathrm{7.4)}$ or acid $(\mathrm{pH} \mathrm{6.8)} \mathrm{medium} \mathrm{for} 6$ hours; then $\mathrm{Na} / \mathrm{H}$ antiporter activity was assayed. Data are plotted as $\mathrm{dpHi} / \mathrm{dt}$, $\mathrm{pH}$ units/min. Controls were cells exposed to transfection reagent without siRNA (No siRNA) or cells transfected with a siRNA against a nonmammalian protein (GL2 siRNA); $n=7$ (No siRNA), $n=6$ (GL2 siRNA), and $n=10$ (pyk2 siRNA). ${ }^{*} P<0.05$ vs. pH 7.4.

nal domains $(14,22)$. The 3 approaches used in our studies were to (a) decrease Pyk2 protein expression with siRNA $\mathrm{pyk}_{2}$, (b) express a dominant-negative $p y k 2$ construct $\left(p y k 2^{\mathrm{K} 457 \mathrm{~A}}\right)$ that removes a key lysine involved in ATP binding, and (c) express the pyk2 $2^{Y 402 F}$ mutant that prevents the SH2 domain in c-Src from binding to its consensus sequence (YAEI) on Pyk2. Our studies show that with all 3 approaches media acidification was unable to stimulate NHE3 activity, demonstrating that Pyk 2 kinase activity is required for acid regulation of NHE3. In addition, these studies show that acid activation of c-Src is mediated by Pyk2.

Having demonstrated that (a) both Pyk2 (present studies) and c-Src $(2,4)$ are required for acid regulation of NHE3 activity, (b) acid incubation increases the abundance of c-Src that coprecipitates with Pyk2, (c) Pyk2/c-Src interaction is required for acid regulation of NHE3 activity, and (d) in the presence of the dominantnegative Pyk $2^{\mathrm{K} 457 \mathrm{~A}}$ acid cannot activate $\mathrm{c}$-Src kinase, we propose a signaling pathway that places $\mathrm{Pyk} 2$ as the direct upstream activator of c-Src. Pyk 2 is generally activated as part of a signaling cascade in response to increases in cell $\mathrm{Ca}^{2+}$, activation of protein kinase $\mathrm{C}$, or activation of $\mathrm{G}$ protein coupled receptors $(7,9)$. Based on its ability to respond to these multiple signals, $\mathrm{Pyk} 2$ is believed to play a role in the response to many extracellular agonists in several different cell types (14). In many systems, Pyk 2 activation is mediated by increases in cell calcium (7). Acid activation of Pyk2 was inhibited in the presence of EGTA, suggesting calcium dependence. However, it should be noted that acid does not elicit a significant increase in cell calcium in OKP cells. Note that our unpublished observations indicate that lowering extracellular $\mathrm{pH}$ from 7.4 to 6.8 results in a small increase in cell $\mathrm{Ca}^{2+}$ from $44.5 \pm 3.8$ to $55.3 \pm 2.6$ $\mathrm{nM}$. The small magnitude of the increase would be atypical for agonists that activate Pyk 2 through changes in cell $\mathrm{Ca}^{2+}$. In addition, there was an unusual time course, with a sharp increase in the Fura-2 340/Fura-2 380 ratio and a constant plateau, suggesting that the small change may be due to a direct effect of $\mathrm{pH}$ on Fura-2 fluorescence. The present studies suggest that intracellular proton concentration also may serve as a regulator of Pyk 2 activity.

The present results demonstrate an important role for Pyk 2 in signaling the effect of decreased intracellular $\mathrm{pH}$ in the renal proximal tubule. Pyk2 is upstream of c-Src and may function as the $\mathrm{pH}$ sensor. Following Pyk 2 activation and autophosphorylation,
c-Src binds to Pyk2 and is activated, with subsequent activation of $\mathrm{p} 125^{\mathrm{FAK}}$ and ERK and increased expression of $c$-fos, c-jun, junB, egr-1, and preproendothelin-1, resulting in NHE3 activation. Espiritu et al. (8) have similarly shown in OK cells that cholinergic agents, angiotensin $\mathrm{II}$, and $\mathrm{CO}_{2}$ activate Pyk2 and that expression of dominant-negative Pyk 2 inhibits activation of $\mathrm{NBC}-1$ by these agonists. In inner medullary collecting duct cells, Pyk 2 and $\mathrm{p} 125^{\mathrm{FAK}}$ are both activated by hypertonicity, with $\mathrm{Pyk} 2$ activation occurring rapidly and $125^{\text {FAK }}$ activation being more delayed (23). The temporal relationship between Pyk2 and p125 $5^{\mathrm{FAK}}$ activation in inner medullary collecting duct cells is similar to that seen with acid incubation of OKP cells (6). Glucocorticoid activation of NHE3 is independent of Pyk 2 and is likely mediated by glucocorticoid receptor-regulated gene expression. The role of Pyk 2 in other acid-induced effects in the kidney and in other cells requires further investigation.

\section{Methods}

Materials. All chemicals were obtained from Sigma-Aldrich with the following exceptions: penicillin and streptomycin (Whittaker MA Bioproducts); acetoxymethyl derivative of BCECF (Molecular Probes); TOPO TA Cloning kit, pcDNA3.1/HIS vector, ThermoScript RT-PCR System, Platinum Taq DNA Polymerase High Fidelity, and dNTP Mix (Invitrogen); pCI-neo, Tfx50, BamHI, and EcoRI (Promega); DNA ligation kit (Takara); cell culture media, Lipofectamine Plus Reagent kits, and Superscript II kit (Gibco BRL); TransIT-TKO siRNA Transfection Reagent (Mirus Corp.); anti-v-Src (Ab-1) monoclonal antibody, NP-40, and protein G-Plus agarose (Calbiochem); goat anti-Pyk2 polyclonal IgG antibody, rabbit anti-c-Src (SRC2) polyclonal IgG antibody, goat and rabbit anti-FAK polyclonal IgG antibodies, rabbit anti-Grb2 polyclonal IgG antibody, and anti-phosphotyrosine antibody (PY99) (Santa Cruz Biotechnology Inc.); rabbit anti-Pyk2 polyclonal IgG antibody (Upstate Biotechnology, Inc.); HRP-labeled anti-rabbit IgG and anti-mouse IgG (Amersham); and ECL kit and ${ }^{32} \mathrm{P}-\gamma$-ATP (PerkinElmer).

Construction of expression vectors for wild-type Pyk2 (pcDNA3.1/HisA/Pyk2) and mutant Pyk2 (pcDNA3.1/HisA/Pyk2 ${ }^{\text {K57A }}$ and pcDNA3.1/HisA/Pyk2 ${ }^{\mathrm{Y} 402 \mathrm{~F}}$ ). The pyk2 cDNA was amplified from Sprague-Dawley rat kidney RNA by PCR with the following composite primers encoding both ends of the rat pyk2 open reading frame flanked with BamHI and EcoRI adapter sequences: 5'-CGGGATCCCGATGTCCGGGGTGTCTGAGC-3' and 5'GGAATTCCTCACTCTGCAGGCGGGTGG-3'.

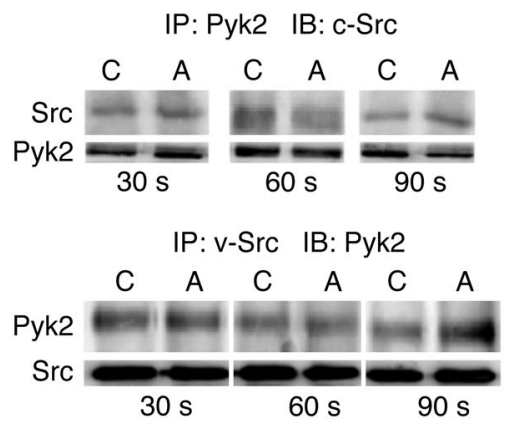

\section{Figure 8}

Acid incubation increases Pyk2 and c-Src coimmunoprecipitation. Representative blots. OKP cells transiently transfected with pyk2 and c-Src were grown to confluence, rendered quiescent, and exposed to control (C) $(\mathrm{pH} \mathrm{7.4)}$ or acid (A) $(\mathrm{pH} \mathrm{6.8)} \mathrm{medium} \mathrm{for} \mathrm{the} \mathrm{indicated} \mathrm{time;} \mathrm{Pyk2} \mathrm{or}$ $\mathrm{v}$-Src was immunoprecipated (IP) as indicated. The immunoprecipitates were divided and immunoblotting (IB) performed using anti-Pyk2 and anti-c-Src antibodies as indicated; $n=3$ for each group. 
A
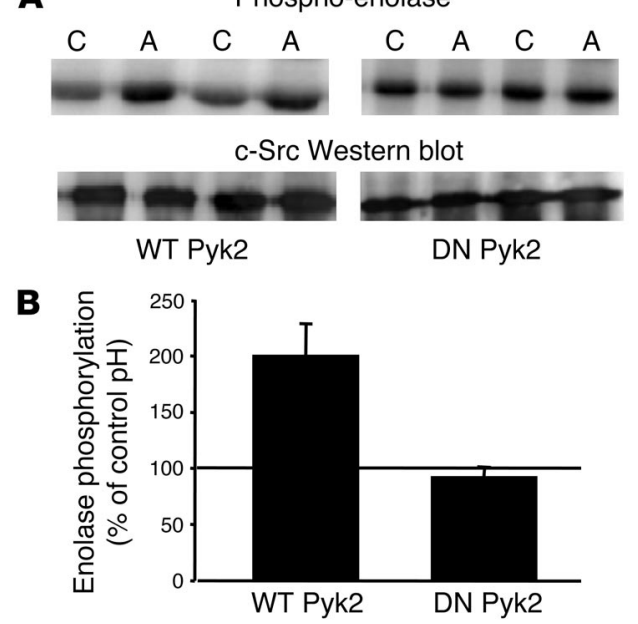

\section{Figure 9}

Pyk2 is required for acid activation of c-Src. OKP cells transiently transfected with wild-type or dominant-negative (DN) pyk2 and wildtype $c$-Src were grown to confluence, rendered quiescent, and exposed to control ( $\mathrm{pH}$ 7.4) or acid ( $\mathrm{pH} \mathrm{6.8)} \mathrm{medium} \mathrm{for} 90$ seconds; c-Src was immunoprecipitated as described in Methods. c-Src kinase activity was assayed using acid-denatured enolase as the substrate. (A) Typical autoradiograph (top) and Western blot (bottom). (B) Summary of the data, which are normalized for C-Src abundance and plotted as percentage of control ( $\mathrm{pH} 7.4) ; n=6$ for studies done with wild-type Pyk2 and $n=4$ for studies done with the dominant-negative Pyk2. c-Src kinase activity was increased in 6 of 6 experiments in the presence of the wild-type Pyk2 and in 1 of 4 experiments in the presence of the dominant-negative Pyk2.

The pyk2 cDNA fragment was cloned into PCR2.1-TOPO vector with a TOPO TA Cloning kit and then subcloned into the pcDNA3.1/HIS vector using BamHI and EcoRI. The DNA sequence was confirmed to be identical to the full-length rat pyk2 sequence in GenBank, with an amino-terminal Xpress-tag.

The kinase-negative, dominant-negative mutants of $p y k 2$, $p y k 2^{\mathrm{K} 457 \mathrm{~A}}$, and pyk2 ${ }^{Y 402 F}$ were generated by replacing lysine 457 with alanine and tyrosine 402 with phenylalanine, respectively, using site-directed mutagenesis, following the manufacturer's protocol (Stratagene). The mutations were confirmed by sequence analysis.

Preparation of OKP pyk2 siRNA. To define the OKP pyk2 sequence, cDNA was prepared from OKP cell total RNA and amplified using the ThermoScript RT-PCR System, according to the manufacturer's protocol. cDNA was amplified with $1 \mathrm{U}$ of Platinum Taq DNA Polymerase High Fidelity for 30 cycles (denaturing at $94^{\circ} \mathrm{C}$ for 30 seconds, annealing at $55^{\circ} \mathrm{C}$ for $30 \mathrm{sec}$ onds, and extension at $68^{\circ} \mathrm{C}$ for 90 seconds). PCR primers were designed from the human $p y k 2$ sequence (GenBank accession number GI1245923) at sites of high homology between human and rat nucleotide sequences. Primers were 5'-GTTGGCTGAGTGCTATGGGCTGA-3' (sense) and 5'TCCTGCATCTGCTTTGGGAAAAA-3' (antisense).

A 21-nucleotide siRNA $A_{\text {pyk2 }}$ duplex with $\mathrm{dTdT}$ overhangs, designed as AA followed by 19 nucleotides of the target OKP mRNA coding sequence (5'-AAUGCCCUUGAUAAGAAGUCC-3') was synthesized, purified, and duplexed by Dharmacon Research.

Cell culture and transfection. OKP cells were passaged in high-glucose (450 mg/dl) DMEM supplemented with 10\% fetal bovine serum, penicillin $(100 \mathrm{U} / \mathrm{ml})$, and streptomycin $(100 \mu \mathrm{g} / \mathrm{ml})(24,25)$. When confluent, cells were rendered quiescent using a 1:1 mixture of low-glucose
(100 mg/dl) DMEM and Ham's F12 in the absence of serum. The length of the quiescent period is indicated in the figure legends.

For DNA transfection, OKP cells were grown to $80-90 \%$ confluence and then were transiently transfected for 5 hours with pcDNA3.1/HisB/LacZ (vector only), pcDNA3.1/HisA/Pyk2 (rat, wild-type pyk2), pcDNA3.1/ HisA/Pyk2 ${ }^{\mathrm{K} 457 \mathrm{~A}}$ (dominant-negative pyk2), or pcDNA3.1/HisA/Pyk2 ${ }^{\mathrm{Y} 402 \mathrm{~F}}$ (c-Src binding deficient) plasmids or cotransfected for 5 hours with wildtype pyk2 (pcDNA3.1/HisA/Pyk2, rat) and wild-type c-Src (pcDNA3/ c-Src, chicken), dominant-negative pyk2 (pcDNA3.1/HisA/Pyk2 ${ }^{\mathrm{K} 457 \mathrm{~A}}$, rat) and wild-type c-Src (pcDNA3/c-Src, chicken), or c-Src binding deficient

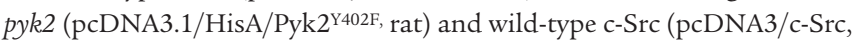
chicken) plasmids using the Lipofectamine Plus kit per manufacturer's instructions. When the transfection medium was removed, cells were incubated in passage medium for 19 hours. At 24 hours after the beginning of transfection, the cells were rendered quiescent.

For siRNA transfection, cells were grown in the same media but without antibiotics 24 hours before transfection. At $50-70 \%$ confluence, siRNA $A_{\text {pyk } 2}$ was transfected into OKP cells using TransIT-TKO siRNA Transfection Reagent, as described by the manufacturer. As a control, siRNA $\mathrm{GL}_{2}$ (corresponding to the firefly luciferase gene) was transfected under similar conditions (12). Other control cells were treated in the same manner but without the addition of siRNA to the transfection reagent. When confluent, cells were rendered quiescent.

To study the effects of media acidification on NHE3 activity, cells were grown on glass coverslips and, after being rendered quiescent, were incubated in control ( $\mathrm{pH} 7.4$ ) or acid ( $\mathrm{pH}$ 6.8) medium for 6 hours (10). For studies examining the effect of medium acidification on c-Src kinase activity or the coprecipitation of Pyk2 and c-Src or Grb2, cells were grown in 60-mm culture dishes and, after being rendered quiescent, were incubated in control $(\mathrm{pH} 7.4)$ or acid ( $\mathrm{pH}$ 6.8) medium for different times, as indicated in the figures. To study the effects of glucocorticoids on NHE3 activity, cells were grown on glass coverslips and, after being rendered quiescent, were incubated in $10^{-7} \mathrm{M}$ dexamethasone or vehicle (ethanol) for 24 hours (11).

$\mathrm{Na}^{+} / \mathrm{H}^{+}$antiporter activity. As previously described, continuous measurement of cytoplasmic $\mathrm{pH}(\mathrm{pHi})$ was accomplished using the intracellularly trapped $\mathrm{pH}$-sensitive dye $\operatorname{BCECF}(10,25)$. To measure $\mathrm{Na}^{+} / \mathrm{H}^{+}$antiporter activity, cells were acidified by addition of nigericin in a $\mathrm{Na}^{+}$-free solution in the absence of $\mathrm{CO}_{2} / \mathrm{HCO}_{3}^{-}$. $\mathrm{Na}^{+} / \mathrm{H}^{+}$antiporter activity was then measured as the initial rate of $\mathrm{pHi}$ increase $(\mathrm{dpHi} / \mathrm{dt})$ in response to $\mathrm{Na}^{+}$addition. Chronic changes in media $\mathrm{pH}$ have no effect on buffer capacity (25). Results are therefore reported as $\mathrm{dpHi} / \mathrm{dt}$.

Immunoprecipitation. Cells were grown to confluence, rendered quiescent, and then washed twice with ice cold PBS; they were lysed with $0.9 \mathrm{ml}$ of icecold RIPA buffer (50 mM Tris-HCl [pH 7.4], $150 \mathrm{mM} \mathrm{NaCl}, 50 \mathrm{mM} \beta$-glycerophosphate, $50 \mathrm{mM} \mathrm{NaF}, 1 \mathrm{mM}$ EGTA, $1 \mathrm{mM} \mathrm{Na}_{3} \mathrm{VO}_{4}, 1 \% \mathrm{NP}-40,0.25 \%$ sodium deoxycholate, $1 \mathrm{mM}$ PMSF, $5 \mu \mathrm{g} / \mathrm{ml}$ aprotinin, $5 \mu \mathrm{g} / \mathrm{ml}$ leupeptin, $2 \mu \mathrm{g} / \mathrm{ml}$ pepstatin). All subsequent manipulations were performed on ice. Cells were scraped with a rubber policeman, rocked for 15 minutes at $4^{\circ} \mathrm{C}$, and centrifuged at $14,000 \mathrm{~g}$ for 15 minutes at $4^{\circ} \mathrm{C}$. The supernatant was diluted to $1 \mathrm{mg}$ protein $/ \mathrm{ml}$, and then incubated with $5 \mu \mathrm{g} / \mathrm{ml}$ goat antiPyk 2 or $1 \mu \mathrm{g} / \mathrm{ml}$ goat anti-FAK polyclonal IgG antibody overnight at $4{ }^{\circ} \mathrm{C}$ with rotation, or with $4 \mu \mathrm{g} / \mathrm{ml}$ mouse $\mathrm{v}$-Src (Ab-1) monocolonal Ab for 1.5 hours at $4{ }^{\circ} \mathrm{C}$ with rotation. Protein G-Plus agarose ( $30 \mu \mathrm{l}$ bead volume) was then added and the mixture incubated for 2 hours at $4^{\circ} \mathrm{C}$ and centrifuged; the pelleted beads were washed 3 times with RIPA buffer.

For coprecipitation studies, cells were grown in 60-mm culture dishes until confluent, rendered quiescent, and incubated in control ( $\mathrm{pH} 7.4)$ or acid ( $\mathrm{pH}$ 6.8) medium for the times indicated in the figures. Pyk2 or c-Src was immunoprecipitated as described above. After having been washed 3 times with RIPA buffer, the pelleted beads were suspended in $60 \mu \mathrm{l} 2 \times$ SDS 
loading buffer and the mixture boiled for 5 minutes. After centrifugation, the supernatant was divided into 2 aliquots, one for immunoblotting with the precipitating antibody and the other for immunoblotting with the nonprecipitating antibody [c-Src (1:500 dilution), Pyk2 (1:1000 dilution), or Grb2 (1:200 dilution)]. The abundance of the coprecipitated protein was normalized to the abundance of the precipitated protein and expressed as a percentage of control ( $\mathrm{pH} 7.4)$.

Immunoblot. Pyk2 or FAK immunoprecipitated from cells grown in $100-\mathrm{mm}$ culture dishes was subjected to $7.5 \%$ SDS-PAGE, and c-Src immunoprecipitated from cells grown in $60-\mathrm{mm}$ culture dishes was subjected to $10 \%$ SDS-PAGE. The samples were then electrophoretically transferred to nitrocellulose. After blocking with blocking buffer ( $5 \%$ nonfat milk and $0.05 \%$ Tween 20 in PBS), blots were probed with a 1:1000 dilution of rabbit anti-Pyk2 IgG antibody, a 1:50 dilution of mouse antiphosphotyrosine antibody, a 1:500 dilution of rabbit antiFAK antibody, or a 1:500 dilution of rabbit anti-c-Src antibody in blocking buffer overnight at $4{ }^{\circ} \mathrm{C}$. Blots were washed 3 times in PBS containing $0.1 \%$ Tween 20 for 10 minutes each and then incubated with a 1:5000 dilution of HRP-labeled anti-rabbit IgG or anti-mouse IgG in blocking buffer for 1 hour and washed as described above. Bands were visualized by ECL and quantified by densitometry.

In vivo phosphorylation and kinase assays. To assay Pyk2 phosphorylation in vivo, confluent, quiescent cells were incubated in control ( $\mathrm{pH} 7.4)$ or acid ( $\mathrm{pH}$ 6.8) medium for the times indicated in the figures. Pyk 2 was immunoprecipitated as described above. After washing 3 times with RIPA buffer, $60 \mu \mathrm{l} 2 \times$ SDS loading buffer was added to the pelleted beads, and the mixture boiled for 5 minutes. The mixture was briefly centrifuged, and the supernatant was divided into 2 aliquots, one for Pyk2 immunoblot to determine Pyk2 abundance (rabbit polyclonal anti-Pyk2 IgG, 1:1000 dilution) and the second for immunoblot with PY99 (1:50 dilution) to measure phosphorylated Pyk2. Results were normalized for Pyk2 abundance and expressed as percentage of control ( $\mathrm{pH}$ 7.4).

To measure c-Src kinase activity in vivo, c-Src was immunoprecipitated as described above from cells grown in $60-\mathrm{mm}$ culture dishes that had been exposed to control ( $\mathrm{pH}$ 7.4) or acid ( $\mathrm{pH}$ 6.8) medium for the times indicated in the figures. The immunoprecipitate was washed 4 times with RIPA buffer and once with Buffer C (20 mM Na HEPES, $10 \mathrm{mM} \mathrm{MgCl}_{2}$, $\mathrm{pH}$ 7.4). The pelleted beads were then suspended in $500 \mu \mathrm{l}$ of Buffer $\mathrm{C}$, divided into 2 aliquots, and pelleted. One aliquot was used to determine c-Src abundance by immunoblot as described above, and the other aliquot was used to assay kinase activity. To assay c-Src kinase activity, the pellet was heated to $30^{\circ} \mathrm{C}$ for 1.5 minutes and then suspended in $20 \mu \mathrm{l}$ of Buffer D (50 mM Na HEPES [pH 7.4], $10 \mathrm{mM} \mathrm{MgCl}, 0.01 \mathrm{mM} \mathrm{ATP,} 0.3 \mathrm{mg} / \mathrm{ml}$ acid-denatured enolase, and $5 \mu \mathrm{Ci}\left[{ }^{32} \mathrm{P}\right]-\gamma$-ATP) at $30^{\circ} \mathrm{C}$ for 10 minutes with rotation. Reactions were stopped by the addition of $20 \mu \mathrm{l}$ of $2 \times$ SDS loading buffer, and the mixture was boiled for 5 minutes. The mixture was then briefly centrifuged, and the supernatant resolved on a 10\% SDS-PAGE gel. Phosphorylated enolase was analyzed by PhosphoImage and quantified by densitometry. Results were normalized for c-Src abundance and expressed as a percentage of control ( $\mathrm{pH}$ 7.4).

In vitro Pyk2 autophosphorylation and Pyk2 and FAK kinase activities. Pyk2 was immunoprecipitated as described above from cells grown in 100-mm culture dishes, and the beads were washed 3 times with RIPA buffer and once with Buffer A (10 $\mathrm{mM} \mathrm{MgCl}_{2}, 10 \mathrm{mM} \mathrm{MnCl}_{2}, 150 \mathrm{mM} \mathrm{NaCl}$ and
$0.2 \mathrm{M}$ Mops) at $\mathrm{pH} 7.4,7.2,7.0$, or 6.8. The pelleted beads were then incubated in $30 \mu \mathrm{l}$ of Buffer A at the respective $\mathrm{pH}$ levels, with $1 \mu \mathrm{g} / \mu \mathrm{l}$ poly(Glu-Tyr) ${ }^{4: 1}, 20 \mu \mathrm{M}$ ATP, and $5 \mu \mathrm{Ci}\left[{ }^{32} \mathrm{P}\right]-\gamma$-ATP for 5 minutes at $32^{\circ} \mathrm{C}$ with rotation. To examine the effect of acid media on the Michaelis-Menten constant for ATP and maximal kinase activity, cold ATP concentrations of $5,20,100$, and $200 \mu \mathrm{M}$ were used with a corresponding amount of hot ATP $(1.25,5,25$, and $50 \mu \mathrm{Ci}$, respectively). To determine whether acid regulation of Pyk 2 was calcium dependent, 1 mM EGTA was added to the reaction buffer. Pyk2 autophosphorylation was studied under the same conditions without the addition of poly(Glu-Tyr $)^{4: 1}$. Reactions were stopped by the addition of $30 \mu \mathrm{l}$ of $2 \times$ SDS loading buffer, and the mixture was boiled for 5 minutes. The mixture was then briefly centrifuged and the supernatant divided into 2 aliquots. One aliquot was used for immunoblot of Pyk2 (rabbit polyclonal anti-Pyk2 IgG, 1:1000 dilution) to determine Pyk2 abundance. The other aliquot was resolved on $15 \%$ (kinase assay) or 7.5\% (autophosphorylation) SDSPAGE gels, analyzed by autoradiography or PhosphoImage, and quantified by densitometry. Results were normalized for Pyk2 abundance and expressed as a percentage of control ( $\mathrm{pH}$ 7.4).

To assay FAK activity, FAK was immunoprecipitated as described above from cells grown in 100-mm culture dishes, and the beads were washed 3 times with RIPA buffer and once with Buffer B $\left(5 \mathrm{mM} \mathrm{MgCl}_{2}, 5 \mathrm{mM}\right.$ $\mathrm{MnCl}_{2}$, and $0.2 \mathrm{M} \mathrm{MOPS}$ ) at $\mathrm{pH} 7.4,7.2,7.0$, or 6.8. The pelleted beads were then incubated in $30 \mu \mathrm{l}$ of Buffer B at the respective $\mathrm{pH}$, with $1 \mu \mathrm{g} / \mu \mathrm{l}$ poly(Glu-Tyr ${ }^{4: 1}, 5 \mu \mathrm{M}$ ATP, and $5 \mu \mathrm{Ci}\left[{ }^{32} \mathrm{P}\right]-\gamma$-ATP for 10 minutes at $25^{\circ} \mathrm{C}$ with rotation. Reactions were stopped by the addition of $30 \mu \mathrm{l}$ of $2 \times$ SDS loading buffer, and the mixture was boiled for 5 minutes. The mixture was then briefly centrifuged and the supernatant divided into two aliquots. One aliquot was used for immunoblot of FAK (rabbit, polyclonal anti-FAK IgG, 1:250 dilution) to determine FAK abundance. The other aliquot was resolved on a $15 \%$ SDS-PAGE gel, analyzed by PhosphoImage, and quantified by densitometry. Results were normalized for FAK abundance and expressed as a percentage of control ( $\mathrm{pH} 7.4)$.

Statistics. Densitometry was performed on a Molecular Dynamics densitometer using the Image Quant program. Statistical analysis was done using a paired or unpaired Student's $t$ test, as appropriate, with significance set at $P<0.05$.

\section{Acknowledgments}

Technical assistance was provided by Ebtesam Abdel-Salam and Carol Wilde. These studies were supported by grants DK39298 and DK20543 from the NIH.

Received for publication February 5, 2003, and accepted in revised form September 14, 2004.

Address correspondence to: Patricia Preisig, University of Texas Southwestern Medical Center, 5323 Harry Hines Boulevard, Room H5.112, Dallas, Texas 75390-8856, USA. Phone: (214) 648-8628; Fax: (214) 648-2071; E-mail: Patricia.Preisig@UTSouthwestern.edu.

Shaoying Li and Soichiro Sato contributed equally to this work.

Patricia Preisig and Robert J. Alpern contributed equally to this work.
1. Alpern, R.J., and Sakhaee, K. 1997. The clinical spectrum of chronic metabolic acidosis: homeostatic mechanisms produce significant morbidity. Am. J. Kid. Dis. 29:291-302.

2. Tsuganezawa, H., et al. 2002. Role of $c$-Src and ERK in acid-induced activation of NHE3. Kidney Int 62:41-50.
3. Yamaji, Y., Moe, O.W., Miller, R.T., and Alpern, R.J. 1994. Acid activation of immediate early genes in renal epithelial cells. J. Clin. Invest. 94:1297-1303.

4. Yamaji, Y., et al. 1995. Overexpression of csk inhibits acid-induced activation of NHE-3. Proc. Natl. Acad. Sci. U. S. A. 92:6274-6278.

5. Yamaji, Y., Tsuganezawa, H., Moe, O.W., and Alp- ern, R.J. 1997. Intracellular acidosis activates c-Src. Am. J. Physiol. 272:C886-C893.

6. Yang, X., Yin, H., Preisig, P.A., and Alpern, R.J. 2000. Acid incubation stimulates $\mathrm{p} 125^{\mathrm{FAK}}$ and paxillin expression and induces stress fiber and focal adhesion formation in OKP cells [abstract]. J. Am. Soc. Nephrol. 11:12A. 
7. Lev, S., et al. 1995. Protein tyrosine kinase PYK2 involved in $\mathrm{Ca}^{2+}$-induced regulation of ion channel and MAP kinase functions. Nature. 376:737-745.

8. Espiritu, D.J.D., Bernardo, A.A., Robey, R.B., and Arruda, J.A.L. 2002. A central role for Pyk2Src interaction in coupling diverse stimuli to increase epithelial NBC activity. Am. J. Physiol. 283:F663-F670.

9. Dikic, I., Tokiwa, G., Lev, S., Courtneidge, S.A., and Schlessinger, J. 1996. A role for Pyk2 and Src in linking G-protein-coupled receptors with MAP kinase activation. Nature. 383:547-550.

10. Amemiya, M., Yamaji, Y., Cano, A., Moe, O.W., and Alpern, R.J. 1995. Acid incubation increases NHE-3 mRNA abundance in OKP cells. Am. J. Physiol. 269:C126-C133.

11. Baum, M., Cano, A., and Alpern, R.J. 1993. Glucocorticoids stimulate $\mathrm{Na} / \mathrm{H}$ antiporter in $\mathrm{OKP}$ cells. Am. J. Physiol. 264:F1027-F1031.

12. Elbashir, S.M., et al. 2001. Duplexes of 21-nucleotide RNAs mediate RNA interference in cultured mammalian cells. Nature. 411:494-498.

13. Bernstein, E., Denli, A.M., and Hannon, G.J. 2001. The rest is silence. RNA. 10:1509-1521.

14. Avraham, H., Park, S.Y., Schinkmann, K., and Avra- ham, S. 2000. RAFTK/Pyk2-mediated cellular signalling. Cell Signal. 12:123-133.

15. Schlaepfer, D.D., and Hunter, T. 1998. Integrin signalling and tyrosine phosphorylation: just the FAKs? Trends Cell Biol. 8:151-157.

16. Nakamura, K., Yano, H., Schaefer, E., and Sabe, H. 2001. Different modes and qualities of tyrosine phosphorylation of Fak and Pyk2 durng epithelial-mesenchymal transdifferentiation and cell migration: analysis of specific phosphorylation events using site-directed antibodies. Oncogene. 20:2626-2635.

17. Blaukat, A., et al. 1999. Adaptor proteins Grb2 and Crk couple Pyk2 with activation of specific mitogen-activated protein kinase cascades. J. Biol. Chem. 274:14893-14901.

18. Laghmani, K., Preisig, P.A., Moe, O.W., Yanagisawa, M., and Alpern, R.J. 2001. Endothelin-1/ endothelin-B receptor-mediated increases in NHE3 activity in chronic metabolic acidosis. J. Clin. Invest. 107:1563-1569.

19. Licht, C., Laghmani, K., Yanagisawa, M., Preisig, P.A., and Alpern, R.J. 2004. An autocrine role for endothelin-1 in the regulation of proximal tubule NHE3. Kidney Int. 65:1320-1326.
20. Gullans, S.R., and Mandel, L.J. 2000. Coupling of energy to transport in proximal and distal nephron. In The kidney: physiology and pathophysiology. D.W. Seldin and G. Giebisch, editors. Lippincott Williams \& Wilkins. Philadelphia, Pennsylvania, USA. 467-482.

21. Soltoff, S.P., and Mandel, L.J. 1984. Active ion transport in the renal proximal tubule, III: The ATP dependence of the Na pump. J. Gen. Physiol. 84:643-662.

22. Park, S.Y., Avraham, H.K., and Avraham, S. 2004 RAFTK/Pyk 2 activation is mediated by trans-acting autophosphorylation in a src-independent manner. J. Biol. Chem. 279:33315-33322.

23. Zhang, Z., Avraham, H., and Cohen, D.M. 1998. Urea and $\mathrm{NaCl}$ differentially regulate FAK and RAFTK/PYK2 in mIMCD3 renal medullary cells. Am. J. Physiol. 275:F447-F451.

24. Cole, J.A., Forte, L.R., Krause, W.J., and Thorne, P.K 1989. Clonal sublines that are morphologically and functionally distinct from parental OK cells. Am. J. Physiol. 256:F672-F679.

25. Cano, A., Preisig, P., and Alpern, R.J. 1993. Cyclic adenosine monophosphate acutely inhibits and chronically stimulates $\mathrm{Na} / \mathrm{H}$ antiporter in $\mathrm{OKP}$ cells. J. Clin. Invest. 92:1632-1638. 